\title{
Pointing Movements on Multi-Layered Displays
}

\author{
J.G. Phillips ${ }^{*}$ and S. Carr
}

School of Psychology, Psychiatry, \& Psychological Medicine, Monash University, Australia

\begin{abstract}
A Multi Layered Display ${ }^{\mathrm{TM}}$ (MLD) uses depth to alter the salience of information. This study considered how the use of depth might influence cursor positioning. As pointing movements to targets may be influenced by flanking nontargets, a MLD was used in an attempt to reduce any distracting effects of non-targets. Participants were 14 skilled computer users who moved a computer cursor using a mouse to virtual targets at two distances in the presence of flanking non-targets on the same or different layer, before or after the target. Cursors avoided the non-targets. Kinematic analysis of cursor trajectories implied less interference in initial cursor trajectories when non-targets were on a different layer. Enhancing target and flanker separation using the MLD reduced the distracting effects of flankers upon the early "planned" part of cursor trajectories. For the latter part of cursor trajectories a MLD may reduce a perceived potential for impact with non-targets.
\end{abstract}

\section{INTRODUCTION}

Operators that are required to navigate complex data structures such as are involved in process control, air traffic control or surveillance, are presented with multiple targets spread across multiple locations, but such tasks may still require some maintenance of situational awareness. Attempts to address cluttered computer screen environments have considered making some screen objects transparent or suggested introducing three-dimensional displays. Threedimensional displays have been proposed to display more information, and improve the salience of selected information [1]. Three-dimensional displays have been reported to improve the ability to visually track multiple targets [2, 3], but it is less clear whether three-dimensional displays can assist target acquisition, namely moving the cursor to a specific target. The present paper evaluated whether advanced screen concepts could increase the perceived separation between targets, and thus reduce any tendencies for non-targets to interfere during cursor positioning movements.

Movement can serve as a metaphor for selection. However non-target objects can influence this selection process. During positioning, non-target objects can attract or repel the reaching movement [4-8]. The time course of target icon acquisition can be influenced by flanking non-targets on computer screens [8], and have been described in [9]. Nontarget objects placed in the line of movement towards the target can influence cursor trajectory. The present study sought to reduce the effects of flanking non-targets by using advanced screen concepts such as transparency and three dimensional displays.

A Multi-Layered Display ${ }^{\text {TM }}$ (MLD) developed by Deep Video Imaging Limited, allows for the manipulation of

*Address correspondence to this author at the School of Psychology, Psychiatry, \& Psychological Medicine, Monash University, Australia; E-mail: jim.phillips@med.monash.edu.au object salience. The $\mathrm{MLD}^{\mathrm{TM}}$ is an LCD screen with two layers separated by $12 \mathrm{~mm}$, that allows viewing of two sources of information simultaneously, one on the front layer and the other on the rear layer. The salience of objects can be varied by placing them before or after the target, and this can be shown to influence movement trajectories [9]. Flankers before the target influence movement trajectories more than flankers after the target. Any effects of flanking non-targets should be reduced when placed on the rear layer of the screen.

\section{METHOD}

Fourteen right handed computer users participated. All participants supplied informed consent in accordance with institutional ethics committee guidelines. The experiment was run on an IBM compatible Intel ${ }^{\circledR}$ Pentium $^{(\mathbb{2}} 4 \mathrm{CPU}$ 2.00G Hz processor desktop computer. The screen was an $18 \mathrm{mxG}$ Multi Layer Display ${ }^{\mathrm{TM}}$ (MLD) from Deep Video Imaging. The MLD ${ }^{\mathrm{TM}}$ comprises two $220 \times 300 \mathrm{~mm}$ LCD layers separated by a distance of $12 \mathrm{~mm}$. Both planes have 24-bit colour depth and a resolution of 1280x1024. All target stimuli appeared on the front layer. Cursor movements were controlled by a Microsoft optical two-button mouse (1.1A USB).

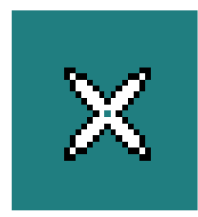

Fig. (1). Crosshair cursor.

The task required participants to use the mouse to move a crosshair cursor (see Fig. 1) on the front layer leftwards or rightwards 150 or $300 \mathrm{~mm}$ from a start location to an $\mathrm{X}$ located within a grey target square. The flanker, when present, was a grey square situated along the line of movement from the start location through the target. 
Flankers were laterally offset $5 \mathrm{~mm}$ before or after the target on the front or rear layer of the screen (see Fig. 2).

Cursor location was sampled as $x, y$ co-ordinates at 200 $\mathrm{Hz}$ (every 5ms). To remove any quantisation error, coordinates were low-pass filtered at $10 \mathrm{~Hz}$. Each condition was presented six times. This was a direction (left, right) by distance (near, far) by flanker (before, after) by the screen layer for the flanker (front, rear) fully repeated measures design. The experiment consisted of one block of 12 practice trials and three blocks of 24 experimental trials.

The dependent variables reflected temporal, kinematic and accuracy characteristics of the movement. Accuracy was reflected by the potential repelling or attracting effect of the flankers, and was indicated by the degree of target over or undershoot, defined as the mean end point of $x$ axis movement (XM). The planning phase of movement was assessed from: reaction time (RT), defined as the time from stimulus presentation to the commencement of cursor motion; and the movement variables of peak velocity $(\mathrm{PkVel})$, time to peak velocity (TTP) and peak acceleration (PkAcc). The terminal guidance phase of movement was assessed from the time from peak to zero velocity.

Data were analysed using separate $2 \times 2 \times 2$ Distance (near, far) by Flanker (before, after) by Layer for flanker (same, different) repeated measures analysis of variance for each dependent variable.

\section{RESULTS}

\subsection{Accuracy}

Flanker effects on the same level should be considered first, and have been described in [9]. As may be seen in Fig. (3a), the accuracy of movements to targets was affected by flanker presence. The flanker tended to repel the cursor. Overall, these results provide evidence for interference in aiming movements when flankers are present but effects depend upon flanker location. When the flanker was on the same level, the effects were only significant for the near target.

The introduction of real depth to the display is achieved by the use of a multi-layer display and is evaluated by presenting target and flankers on the same or different layer. The effect of layer examines the effect of depth upon the targeting task when performed in the presence of flankers. The presence of the flankers influenced movement endpoint location, and these effects were modified by layer and distance. There was a significant layer by flanker by distance interaction, $F(1,13)=7.742, p<0.05, f=0.772$. The interaction was decomposed into two layer by flanker repeated measures ANOVAs, one for the 'near' target and one for the 'far' target.

For the near target location, there were no differences in accuracy performance between layers $(F(1,13)=3.299, p>$ $0.05, f=0.449$ ), and accuracy did not change as a function of layer and flanker position $(F(1,13)=2.426, p>0.05, f=$ $0.547)$. There was a significant change in performance for flanker location. When the flanker was before the target there was significantly more overshoot, $(M=1.73 \mathrm{~mm})$ than when the flanker was after the target $(M=0.60 \mathrm{~mm}), F(1,13)$ $=20.564, p<0.05, f=0.954$ on both layers. This result implies the flankers, even though separated by depth, continue to activate attentional mechanisms that lead to the flankers repelling movement.
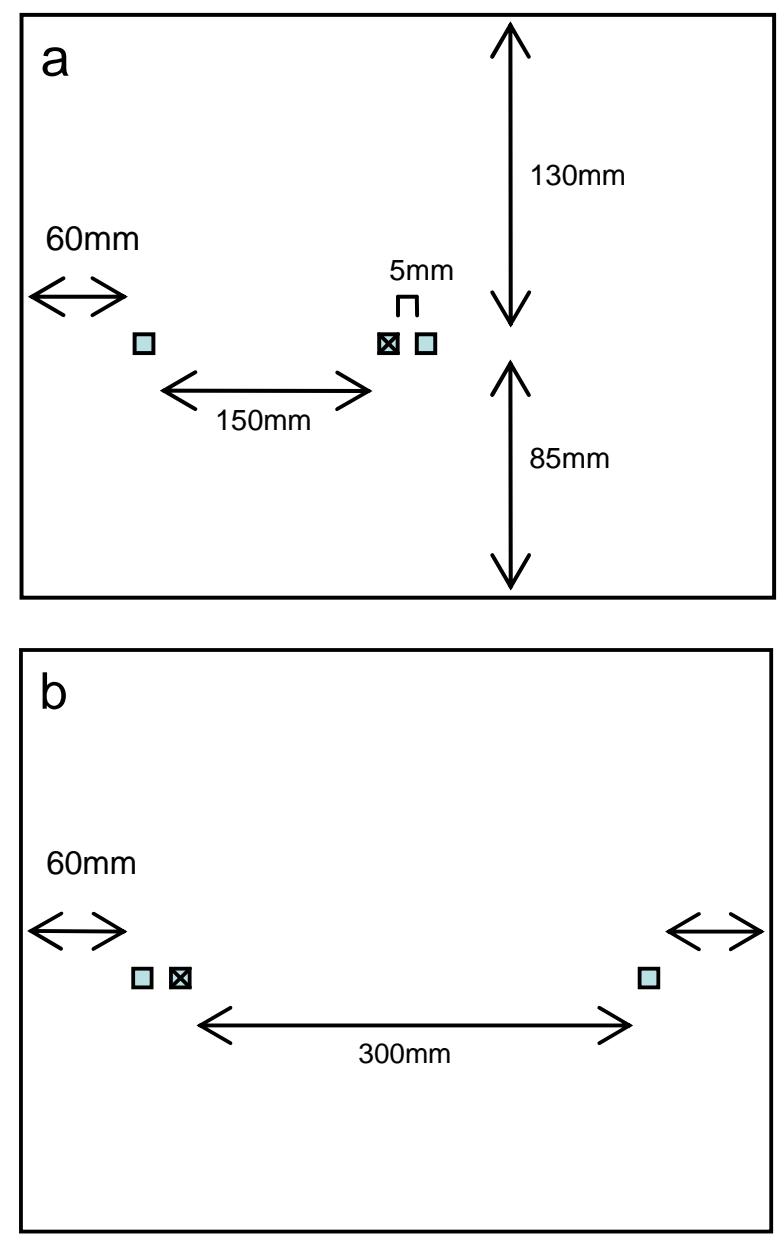

Fig. (2). Experimental Stimuli. Targets contain an X. Fig. (2a) denotes a left start to a near target. Fig. (2b) denotes a right start to a far target.

For the far target location, the analysis of layer and flanker effects revealed a different pattern of effects. The flanker effect changed as a function of screen layer, $F(1,13)$ $=5.618, p<0.05, f=0.437$. This interaction was decomposed into four one way ANOVAs. There was a significant difference in accuracy performance on the different layer, with significantly more overshoot when the flanker was before the target $(M=1.14 \mathrm{~mm})$ compared to $\operatorname{after}(M=0.06 \mathrm{~mm})(F(1,13)=16.774, p<0.05, f=1.368)$. This effect was not found when the flankers were on the same layer $(F(1,13)=0.553, p>0.05, f=0.127)$. When the flanker was on a different level, as may be seen in Fig. (3b), the flanker was repelling the cursor for near and far targets. The MLD did not eliminate these flanker effects. On the different layer, there was also significantly more overshoot when the flanker was before the target $(M=1.14 \mathrm{~mm})$ compared to the flanker before on the same layer $(M=$ $0.72 \mathrm{~mm}), F(1,13)=5.141, p<0.05, f=0.411$. This effect was not found for the flankers after the target, $F(1,13)=$ $1.176, p>0.05, f=0.361$. 


\subsection{Planned Phase}

The phases of movement that are associated with planning are reaction time and aspects of the initial movement trajectory [5-7]. There were no significant effects for reaction time or peak velocity. However a flanker before the target reduced peak acceleration.

Peak acceleration can be viewed as a measure of the initial programmed phase of movement [5-7] and there was a significant effect of the flankers. Peak acceleration was significantly less with the flanker before $\left(M=4261.49 \mathrm{msec}^{-2}\right)$ than with the flanker after $\left(M=4466.07 \mathrm{msec}^{-2}\right)$ the target $(F(1,13)=9.272, p<0.05, f=0.945)$. If interference can be viewed as a slowing of peak acceleration, then this interference was reduced by using depth. Peak acceleration was greater when the flanker was on a different layer $\left(M=4435.97 \mathrm{msec}^{-2}\right)$ than when the flanker was on the same layer $\left(M=4291.6 \mathrm{msec}^{-}\right.$ 2) $(F(1,13)=7.035, p<0.05, f=0.945)$. There were no significant interactions. These results demonstrate less interference in the initial phases of movement performance when the flankers were separated by depth, however the presence of a flanker in any condition was still enough to cause some interference.

As might be expected peak acceleration was greater when movements of greater extent were required with movements to the far $\left(M=5005.92 \mathrm{msec}^{-2}\right)$ target having greater peak acceleration than movements to the near $\left(M=3721.64 \mathrm{msec}^{-2}\right)$ target $(F(1,13)=18.046, p<0.05, f=1.178)$.

\subsection{Terminal Guidance Phase}

Interference in the terminal guidance phase of movement is characterised by slower times from peak to zero velocity. There was no layer by flanker by distance interaction $(F(1,13)=0.644$, $p>0.05, f=0.222$ ), however time spent in terminal guidance did change as a function of flanker location and distance for both layers $(F(1,13)=7.202, p<0.05, f=2.684)$ (see Fig. 4). In near space it appears that terminal aspects of the movement are foreshortened or extended in an attempt to "dodge" the flanker. In far space on the same layer, the flankers have no effect, but on the different layer, the flankers may be capturing users' attention.

(a)

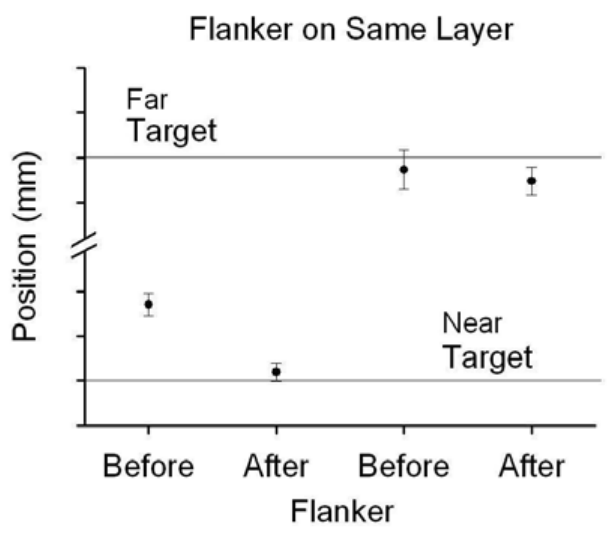

\section{DISCUSSION}

Multi-Layer displays (MLD) use variations in depth to enhance the salience of key elements in cluttered computer screens while maintaining some degree of situational awareness. Most studies consider whether the MLD can use the top layer to improve the salience of items within a multi-element display during tracking tasks. The present study considered whether MLD can reduce interference caused by flanking objects during reaching movements. Flanking non-targets can influence the acquisition of target icons on computer screens [8]. This study considered whether the level of interference caused by nontarget objects during cursor positioning [9] could be reduced by separation in depth afforded by a MLD.

The presence of a flanking non-target tended to repel movement. Interference caused by a flanking non-target before the target manifested as reduced peak acceleration. The depth provided by the MLD seemed to reduce the interference associated with flanking non-targets by increasing peak acceleration. Nevertheless, during terminal guidance, the depth afforded by the MLD appeared to increase interference, exacerbating flanker effects. The mixed findings in the present study may be due to the action of some other variable such as opacity of screen objects [2], or it may be because the $12 \mathrm{~mm}$ separation employed by the MLD was insufficient [10].

Separation of targets in depth may assist the tracking of targets [3], but may potentially influence aiming or target acquisition. In the present study the continued interference of the flankers implies the separation of objects by depth does not preclude attentional mechanisms of non-target inhibition from occurring [see 11]. In light of everyday reaching, this result is not surprising. For instance, when reaching for a glass on a table, glasses that surround one's own glass are separated by depth, however reaching continues to take account of non-target glasses [4]. When those glasses are attended to in the action centred reference frame, they are processed to initiation stage and subsequently inhibited.

Dissociations between the attentional components associated with planning and the motoric processes associated with execution should not be surprising as they are likely to involve separate mechanisms. For instance

(b)

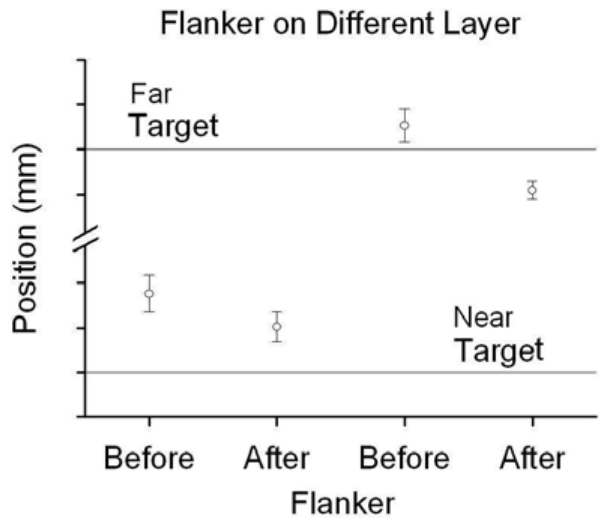

Fig. (3). Effects of flanker on movement endpoints for (a) same and (b) different layers. Target locations are denoted by horizontal lines. Solid vertical bars represents mean end points of movement $(\mathrm{mm})$ with respect to the target, error bars represent one standard error. Flanker and target size and locations are not to scale. 
during choice reaction time tasks Brewer [12] observed that the number of alternatives had a greater influence upon reaction time than movement duration. Conversely, Fitts and Petersen [13] observed during positioning tasks that movement difficulty predominantly affected movement duration but not reaction times.

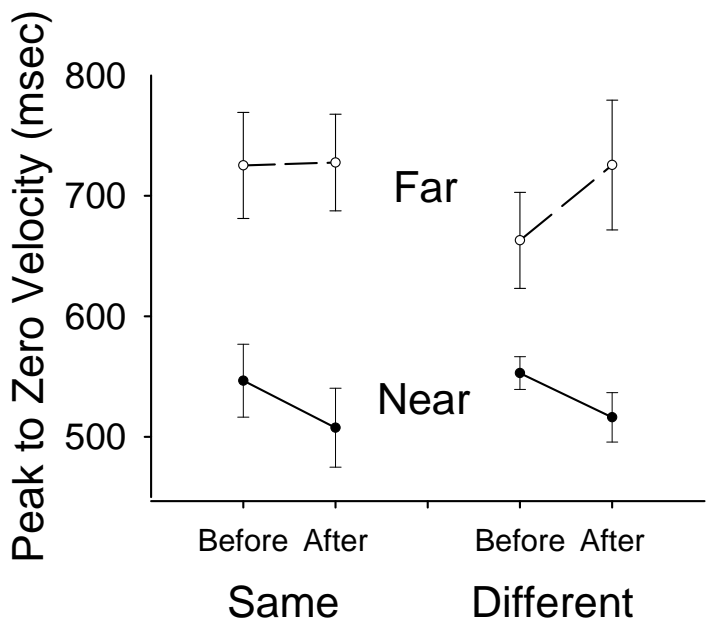

Fig. (4). Effect of flanker position and target distance for time from peak to zero velocity $(\mathrm{msec})$ for same and different layers. Figures represent means and error bars indicate the standard error.

Milner and Goodale [14] have proposed different visual pathways are employed for object recognition and perception for action. On this basis depth could perhaps alter object salience, but not object recognition. Indeed the perceptual constancies serve to maintain recognisability of objects despite changes in depth or orientation [15]. Nevertheless, variations in depth have clearer implications for computation of movement trajectories in terms of aiming point or amounts of acceleration required. This perhaps explains why the initial acceleration of cursor motion is enhanced when the flanker is separated in depth from the target. Under such circumstances the flanker is less likely to be viewed as an obstacle.

The potential for interference observed for far targets on different layers is less explicable as research has focussed upon the early parts of the movement trajectory [4-7]. As a cursor controller, the mouse can be prone to overshoot when movements of greater extent are required [16]. In addition, trajectories do vary as a function of whether they terminate with impact or not [17]. The MLD appeared to increase the separation associated with potentially obstructive objects in far space. Hence there could have been less perceived potential for impact, and this may have had implications for braking forces as participants would no longer have to be as careful with their points of aim.

\section{CONCLUSION}

The present data implies that an "aiming spot" is modulated (possibly throughout the time course of the movement) to avoid distracting objects. The depth afforded by the MLD possibly reduces interference in near space, where movements might still be programmed. However the perceived separation may actually reduce the care taken during the decelerative terminal guidance phase when larger full screen movements are required.

\section{ACKNOWLEDGEMENTS}

The research was facilitated by the loan of apparatus from the Defence Science and Technology Organisation. The authors gratefully acknowledge the input of Dr. James Meehan.

\section{REFERENCES}

[1] Kooi FL. The case for transparent depth displays. Report TNO-TM 2001-A067/E, Soesterberg, The Netherlands: TNO Human Factors 2001.

[2] Viswanathan L, Mingolla E. Dynamics of attention in depth: evidence from multi-element tracking. Percept 2002; 31: 1415-37.

[3] Dünser A, Billinghurst M, Mancero G. Evaluating visual search performance with a multi layer display. Proceedings of OZCHI, Cairns. December 8-12 2008; Available from: http://portal.acm. org/citation. $\mathrm{cfm}$ ?id=1517744.1517796\&coll=portal\&dl=ACM

[4] Tipper SP, Howard LA, Jackson SR. Selective reaching to grasp: evidence for distractor interference effects. Vis Cognit 1997; 4: 138.

[5] Welsh TN, Elliott D. Effects of response priming and inhibition on movement planning and execution. J Mot Behav 2004; 36: 200-11.

[6] Welsh TN, Elliott D. Movement trajectories in the presence of a distracting stimulus: evidence for a response activation model of selective reaching. Q J Exp Psychol 2004; 57(A): 1031-57.

[7] Welsh TN, Elliott D. The effects of response priming on the planning and execution of goal-directed movements in the presence of a distracting stimulus. Acta Psychol 2005; 119: 123-42.

[8] Lyons J, Elliott D, Ricker KL, Weeks TN, Chua R. Action-centred attention in virtual environments. Can J Exp Psychol 1999; 53: 176-87.

[9] Carr SH, Phillips JG, Meehan JW. Non-target flanker effects on movement in a virtual action centred reference frame in Graphical User Interfaces. Exp Brain Res 2008; 184: 95-103.

[10] Andersen GJ, Kramer AF. Limits of focused attention in 3dimensional space. Percept Psychophys 1993; 53: 658-67.

[11] Atchley P, Kramer AF. Object and space-based attentional selection in three-dimensional space. Vis Cognit 2001; 8: 1-32.

[12] Brewer $N$. Intellectual retardation and parameters of choice reaction time. Unpublished doctoral dissertation. South Australia: University of Adelaide 1976.

[13] Fitts PM, Peterson JR. Information capacity of discrete motor responses. J Exp Psychol 1964; 67: 103-12.

[14] Milner AD, Goodale MA. The visual brain in action. Oxford, England: Oxford University Press 1995.

[15] Day RH. Human perception. Sydney: John Wiley \& Sons 1969.

[16] Phillips JG, Triggs TJ. Characteristics of cursor trajectories controlled by the computer mouse. Ergonomics 2001; 44: 527-36.

[17] Teasdale N, Schmidt RA. Deceleration requirements and the control of pointing movements. J Mot Behav 1991; 23: 131-8. 